\title{
Ophthalmic and molecular genetic findings in Kniest dysplasia
}

PI Sergouniotis' ${ }^{1}$ GS Fincham ${ }^{1}$, AM McNinch ${ }^{1,2}$, C Spickett ${ }^{2}$, AV Poulson ${ }^{1}$, AJ Richards ${ }^{1,2}$ and MP Snead ${ }^{1}$

\begin{abstract}
Purpose To study the variability of the ophthalmic phenotype in Kniest dysplasia. Kniest dysplasia is an inherited disorder associated with defects in type II collagen and characterised by short-trunked dwarfism, kyphoscoliosis, and enlarged joints with restricted mobility. Other features include marked hand arthropathy, cleft palate, hearing loss, and ocular abnormalities (myopia, abnormal vitreous, and high risk of developing retinal detachment).

Methods Data from eight unrelated individuals with a clinical and molecular diagnosis of Kniest dysplasia are reported. Clinical assessment included an audiogram and ophthalmological examination in all but one patient who died in the immediate postnatal period. Sanger sequencing of the COL2A1 gene was performed.
\end{abstract}

Results Six of the seven patients tested were high myopes with one patient being an emmetrope. Bilateral quandratic cataracts and subluxed lenses were noted in one subject. Variable but abnormal vitreous architecture was observed in all seven individuals tested. Six of the seven patients had significant hearing impairment and five of the seven patients exhibited clefting abnormalities. One patient had bilateral retinal detachments in his twenties. Six dominant disease-causing COL2A1 variants were detected. In three cases, testing of parental samples revealed that the disease-causing variant was not present in either parent.

Conclusion The ophthalmic features in Kniest dysplasia are very similar to those in other disorders of type II collagen such as Stickler syndrome. It is likely that different type II collagenopathies have a similar level of ocular morbidity and regular ophthalmologic examination is recommended. Kniest dysplasia is associated with heterozygous $C O L 2 A 1$ mutations that are frequently de novo.

Eye (2015) 29, 475-482; doi:10.1038/eye.2014.334; published online 16 January 2015

\section{Introduction}

All collagen molecules are composed of a triple helix consisting of three $\alpha$-chains. In the case of type II collagen, which is the major structural component of cartilaginous tissues and the vitreous, these chains are identical (forming a homotrimer) and are encoded by the COL2A1 gene (MIM + 120140). ${ }^{1,2}$ Defects in this gene give rise to a phenotypically diverse group of disorders collectively termed type II collagenopathies (or COL2A1-related disorders). ${ }^{3}$ These conditions are typically inherited in an autosomal dominant manner and present with a multisystem phenotype that may include skeletal, orofacial, auditory, and ocular features. Notably, type II collagenopathies span a wide range of clinical severity ranging from 'ocular-only' variants of Stickler syndrome (MIM \#609508) ${ }^{2}$ to perinatally lethal chondrodysplasias (for example, hypochondrogenesis (MIM \#200610)) ${ }^{4}$ In the middle of this spectrum lies a subgroup of disorders presenting with short stature at birth. The differential diagnosis of such type II collagenopathies causing short stature includes spondyloepiphyseal dysplasia congenita (SEDC (MIM \#183900)), spondyloepimetaphyseal dysplasia (SEMD Strudwick type (MIM \#184250)), spondyloperipheral dysplasia (MIM \#271700), and Kniest dysplasia (MIM \#156550).

Kniest dysplasia is a dominantly inherited COL2A1-related disorder, first described by Wilhelm Kniest in 1952. ${ }^{3,5}$ Classically, it is characterised by short-trunked dwarfism (expected height 100-140 cm; http://www.ksginfo.org/aboutus.html,
${ }^{1}$ Vitreoretinal Service, Addenbrooke's Hospital, Cambridge University NHS Foundation Trust, Cambridge, UK

${ }^{2}$ Department of Pathology, University of Cambridge, Cambridge, UK

Correspondence: MP Snead, Vitreoretinal Service, Addenbrooke's Hospital, Cambridge University NHS Foundation Trust, BOX 41, Hills Road, Cambridge CB2 2QQ, UK Tel: +44 (0)12 23216701 ; Fax: +44 (0)12 23217968 . E-mail: mps34@cam.ac.uk

Received: 1 June 2014 Accepted in revised form: 9 December 2014 Published online: 16 January 2015 
accessed on 27 September 2014), kyphoscoliosis, and short limbs with prominent joints that restrict mobility. 6 Major problems arise from progressive joint enlargement and contracture, especially affecting the metacarpophalangeal and interphalangeal joints. ${ }^{2}$ Additional associated features of the syndrome, including orofacial abnormalities (cleft palate, small jaw, and flat mid-face), hearing loss (conductive, sensorineural, or mixed), and ocular abnormalities, are shared with the other type II collagenopathies. ${ }^{4}$ In a case series of seven patients clinically diagnosed with Kniest dysplasia, Maumenee and Traboulsi ${ }^{7}$ advocated the need for ophthalmological assessment in all individuals with the disorder; they concluded that congenital nonprogressive high myopia, abnormal vitreous on slit lamp examination, and an increased risk of retinal detachment are important features of the syndrome.

Herein, we report the findings in eight patients with a clinical diagnosis and molecular confirmation of Kniest dysplasia. Variability in the ophthalmic phenotype is described and COL2A1 allelic heterogeneity is discussed.

\section{Materials and methods}

Eight individuals (median age: 11 years old) with clinical and radiographic features of Kniest dysplasia (including short stature and prominent joints with reduced mobility) and heterozygous likely disease-causing variants in COL2A1 were ascertained from the Vitreoretinal Service at Addenbrooke's Hospital, Cambridge, UK. The local research ethics committee approved the study, and all investigations were conducted in accordance with the principles of the Declaration of Helsinki; informed consent was obtained from all participating individuals. Six of the cases have been partially described in previous reports (Table 1).8,9

Clinical assessment included a detailed history and palatal, audiological, and ophthalmological examination in all but one patient who died in the immediate postnatal period owing to respiratory distress associated with the condition (subject KN1). Cycloplegic refraction was undertaken in two patients (subjects KN2 and KN3; aged 4 and 7 years, respectively) and subjective refraction was performed in four patients (subjects KN4-KN7; aged 11-29 years). Vitreous architecture was assessed on slit lamp biomicroscopy by at least one clinician (MPS) in seven of the subjects studied (it was not possible to test subject $\mathrm{KN} 1$ ).

Screening of the COL2A1 gene was performed according to previously described methods; ${ }^{10}$ all eight patients and, where possible, parental DNA samples were tested. Genomic DNA was extracted from the peripheral blood lymphocytes of the donated blood samples. The COL2A1 coding region and intron-exon boundaries of exons 1-54 (NCBI reference sequence NG_008072.1; transcript NM_001844.4) were amplified and sequenced. To predict whether the missense changes associated with Kniest dysplasia affect exonic splice regulatory sequences and/or generate a cryptic splice site within COL2A1, the wild-type and mutant sequences of the corresponding exons were analysed using Alamut version 2.3 (Interactive Biosoftware, Rouen, France, accessed on 31 May 2014). The splicing prediction tools within Alamut include MaxEntScan (http://genes. mit.edu/burgelab/maxent/Xmaxentscan_scoreseq. html), NNsplice (http:/ /www.fruitfly.org/seq_tools/ splice.html), human splicing finder (http:/ / www.umd. be/HSF/), ESE finder (http://rulai.cshl.edu/cgi-bin/ tools/ESE3/esefinder.cgi?process=home), and RescueESE (http://genes.mit.edu/burgelab/rescue-ese/). All variants are described according to the Human Genome Variation Society (HGVS) guidelines based on reference sequence NM_001844.4 (COL2A1).

\section{Results}

The clinical features and COL2A1 screening results of the eight study participants are summarised in Table 1. Patients were examined by an ophthalmologist within the first year of life. Typical skeletal manifestations and radiographic features were observed in all the cases (Figure 1). Five of the seven tested patients exhibited palatal clefting abnormalities that required formal surgical repair; six of the seven patients had significant hearing impairment, which was either due to conductive or sensorineural loss, or a combination of both.

Visual acuity ranged from -0.100 to +2.000 logarithm of the minimum angle of resolution (LogMAR) units (median $+0.175)$. Six of the seven tested patients were high myopes; the documented refractive error ranged from plano to - 14.50 dioptres (median spherical equivalent refraction - 11.00 dioptres). Low astigmatism was observed in most cases, with the highest identified cylindrical correction being 3.00 dioptres. Bilateral quadratic lamellar lens opacities, in addition to bilateral inferiorly subluxed lenses, were noted in one patient (subject KN4).

Abnormal vitreous architecture, visible on slit lamp biomicroscopy, was documented in all the seven examined patients. In five cases this was consistent with a membranous vitreous anomaly in the immediate retrolental space (identical to that seen in type I Stickler syndrome) ${ }_{i}^{11}$ the remaining two cases had a hypoplastic vitreous with reduced lamellae and a fibrillar cortex. One patient (subject KN8) had bilateral rhegmatogenous retinal detachments at ages 26 and 27 . The right eye had a macula-on detachment and was treated with a $360^{\circ}$ buckle and laser retinopexy (acuity after surgery 0.175 LogMAR), whereas the left eye had recurrent 


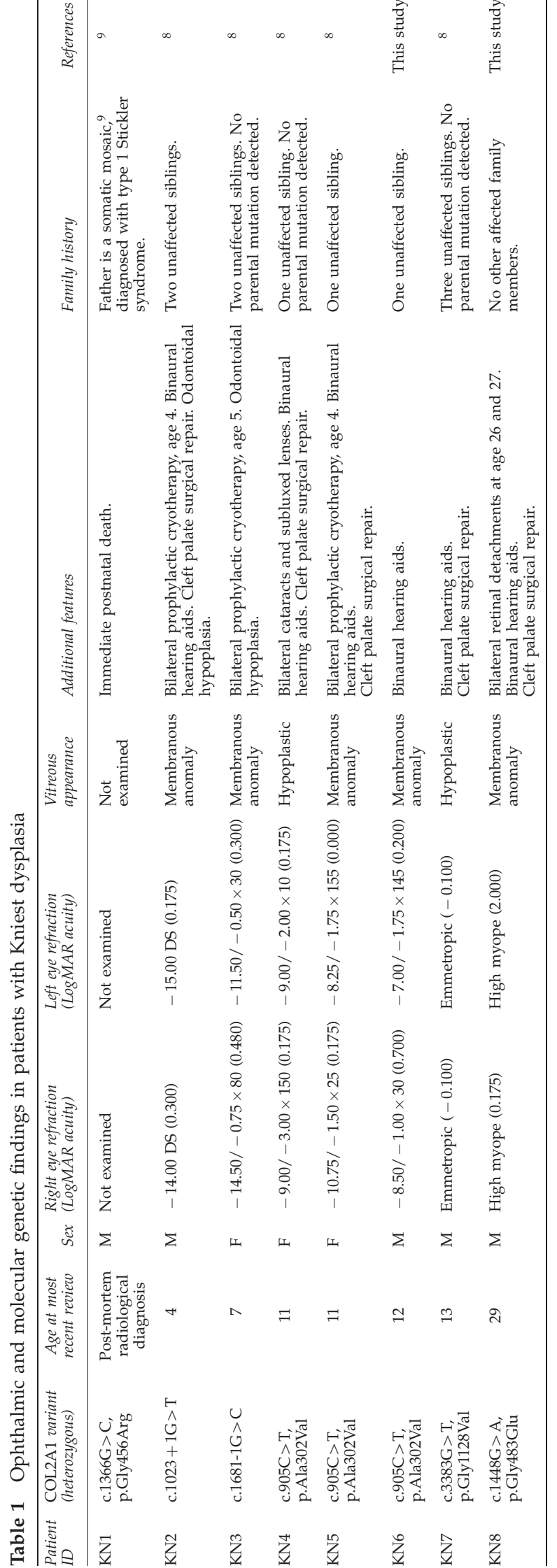

detachments that were complicated by proliferative vitreoretinopathy; there was silicone oil in situ in his left pseudophakic eye and the left acuity was 2.000 LogMAR. None of the other examined study subjects (mean age 11) had a retinal break at presentation, and the posterior hyaloid membrane was attached in all their eyes.

Three patients had bilateral $360^{\circ}$ prophylactic cryoretinopexy according to a standardised protocol (http://www.vitreoretinalservice.org/Prophylactic CryotherapyProtocol.html, accessed on 27 September 2014). This protocol was designed to prevent the retinal detachment secondary to giant retinal tears and has been previously shown to be safe and to substantially reduce (but not eliminate) the risk of detachment in individuals with type I Stickler syndrome. ${ }^{12}$

Six different likely disease-causing DNA variants in COL2A1 were detected in eight alleles of the eight patients. These included four missense (c.1366G $>$ C, p.Gly456Arg; c.1448G > A, p.Gly483Glu; c.3383G > T, p.Gly1128Val, and c.905C $>$ T, p.Ala302Val) and two splice site (c.1023 + 1G > T and c.1681-1G >C) changes (Tables 1 and 2). In three cases, parental testing revealed the disease-causing variant to be not present in either parent (subjects KN3, KN4, and KN7). The father of subject KN1 was a somatic mosaic for the c.1366G > C, p.Gly456Arg mutation and had an existing diagnosis of type I Stickler syndrome, as previously reported. ${ }^{9}$ Parental samples were not available for segregation analysis in the four remaining study subjects (KN2, KN5, $\mathrm{KN} 6$, and KN8).

\section{Discussion}

To date, over 400 likely disease-causing variants have been described in the COL2A1 gene (HGMD, Human Gene Disease Mutation Database, http://www.hgmd. cf.ac.uk/, accessed on 27 September 2014). Of these, 25 have been associated with Kniest dysplasia (Table 2). Here, we discuss allelic heterogeneity in Kniest dysplasia and report the ophthalmic phenotype in this type II collagenopathy.

The diagnosis of a type II collagenopathy has important implications as it can highlight the existing problems that have been overlooked and allow the prediction of future complications. On the basis of the severity of skeletal, radiographic, and extraskeletal manifestations, type II collagenopathies have been classically viewed as a mixture of distinct conditions (for example, Stickler syndrome, SEDC, and Kniest dysplasia). Such categorisation often has no obvious mechanistic basis and is often confounded by the phenotypic variability (family of subject $\mathrm{KN} 1^{9,13}$ ) and by the presence of age-dependent phenotypic transitions. ${ }^{13-15}$ Therefore, clinical suspicion for all 


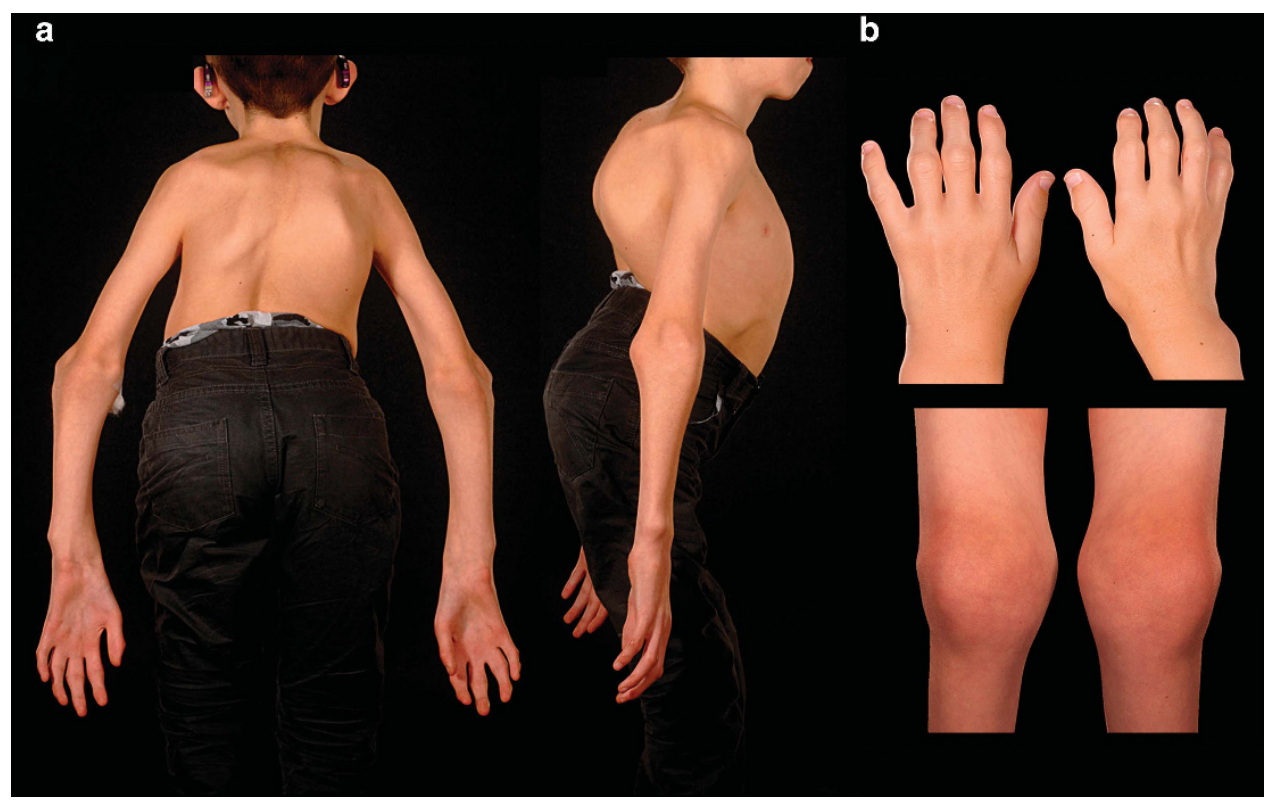

Figure 1 Colour photos of subject KN6 (a) and subject KN2 (b) showing kyphoscoliosis, short trunk, prominent large joins, flexion contractures of hips and fingers, and interphalangeal joint enlargement.

manifestations of type II collagenopathies is advised when managing cases of Kniest dysplasia. Some relatively common and important systemic features of the disorder identified here and elsewhere include a narrow chest (subject $\mathrm{KN1}{ }^{16,17}$ ), laryngotracheomalacia (subject $\mathrm{KN}^{18}{ }^{18}$ ), odontoidal hypoplasia resulting in cervical instability (subjects KN4 and KN5 $5^{19,20}$ ), and hearing impairment (subject KN2, KN3, KN5, KN6, KN7, and $\mathrm{KN} 8^{8}$ ).

All examined patients in the present cohort had congenitally abnormal vitreous gel architecture; five of the seven patients exhibited the membranous type 1 vitreous anomaly seen in type I Stickler syndrome. Notably, although abnormal vitreous architecture appears to be a consistent finding among type II collagenopathies, cases with normal vitreous have previously been reported. ${ }^{8}$ Myopia was present in six of the seven examined patients in this series, with the remaining study subject being found to be emmetropic (subject KN7). This reinforces the notion that although congenital myopia is an important feature of COL2A1related disease, it is not a reliable screening tool for exclusion. ${ }^{2}$ In addition to abnormal vitreous and myopia, patients with Kniest dysplasia have been reported to have a high risk of developing rhegmatogenous retinal detachment. ${ }^{7}$ Maumenee and Traboulsi ${ }^{7}$ reported the occurrence of retinal detachment (during the first or second decade of life) in four of seven cases with Kniest dysplasia. None of the participants in this study had suffered a retinal detachment during their childhood, but one case (subject KN8) developed retinal detachments in his twenties. Although a case of retinal dialysis has been previously reported in a patient with Kniest dysplasia, given the similarities in the vitreoretinal phenotype with other type II collagenopathies (including type I Stickler syndrome), we speculate that retinal tears are more likely to be the main cause of detachment in these patients. ${ }^{2,21}$ Therefore, after informed consent, three study subjects had prophylactic retinopexy designed and positioned to prevent retinal detachment arising from giant retinal tears. Importantly, the efficacy of such an approach has not been demonstrated in Kniest dysplasia but a significant reduction in the risk of retinal detachment has been shown in type I Stickler syndrome. ${ }^{12}$

Most mutations associated with Kniest dysplasia are in-frame deletions and/or presumed to disrupt normal COL2A1 pre-mRNA splicing (Table 2). Notably, six missense changes have been previously reported (c.905C > T, p.Ala302Val; c.908G > A, p.Gly303Asp; c.980G > A, p.Gly327Asp; c.1366G > C, p.Gly456Arg; c.1375G > C, p.Gly459Arg; c.3383G > T, p.Gly1128Val). The c.905C > T, p.Ala302Val change, which appears to be the commonest cause of Kniest dysplasia in both the present cohort and the literature (Table 2), has been previously shown to create a cryptic splice-donor site within exon $14 .^{22,23}$ The effect of the remaining five missense changes on splicing has not been studied to date; we have used online bioinformatics tools to make predictions and the results are presented in Table 2. It is not uncommon in human disease that coding point 


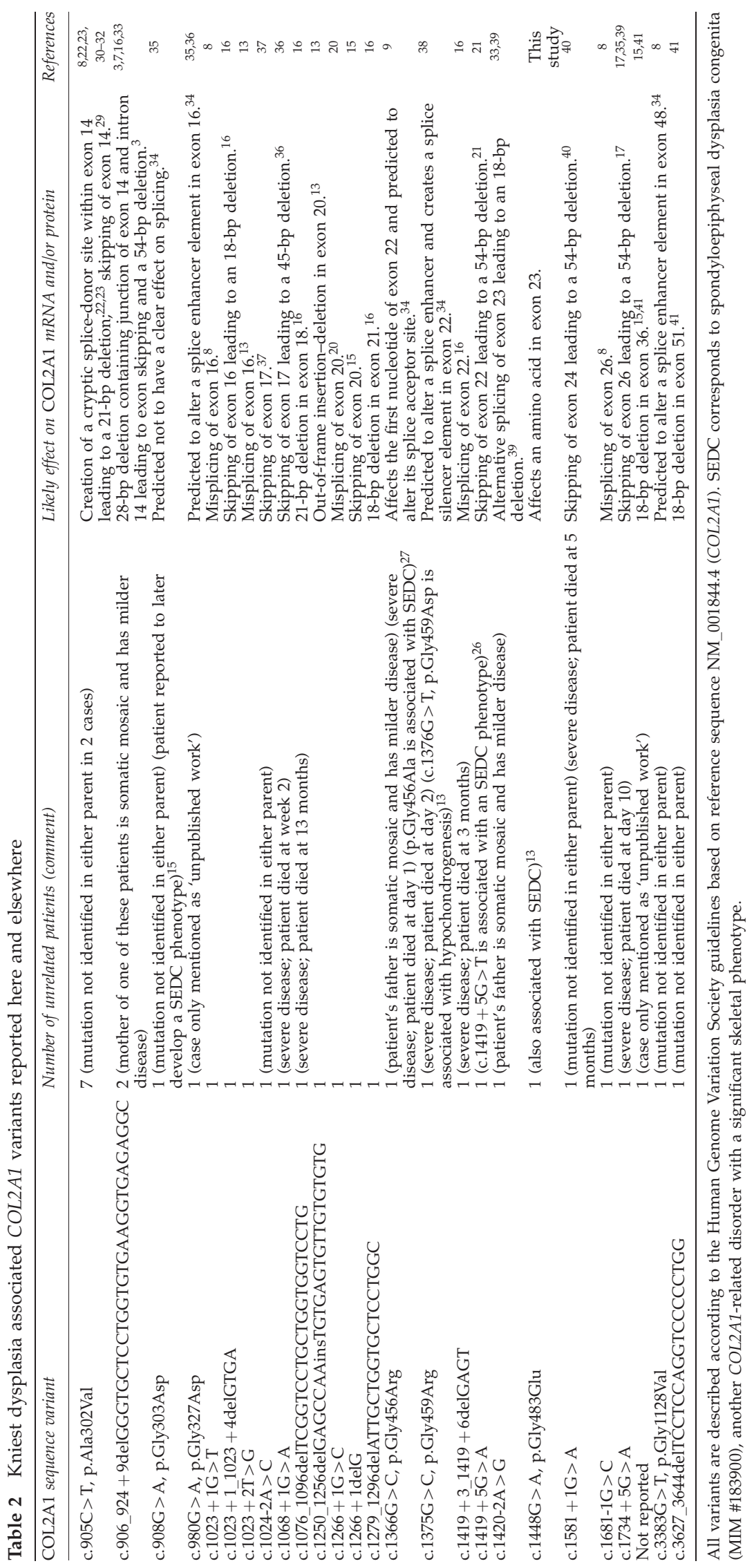


mutations have an unexpected effect on splicing, ${ }^{24,25}$ and mRNA experiments are expected to provide further insight.

The p.Gly483Glu change identified in subject KN8, a 29-year-old male with radiographic findings consistent with Kniest dysplasia, has not been previously associated with the condition but was reported in a patient with SEDC. ${ }^{13}$ It is of interest that the base pair substitutions of c.1419+5G have also been associated with both Kniest dysplasia (c. $1419+5 \mathrm{G}>\mathrm{A})^{21}$ and SEDC (c. $1419+5 \mathrm{G}>\mathrm{T}){ }^{26}$ Furthermore, amino-acid substitutions of p.Gly456 and p.Gly459 have been reported in cases of Kniest dysplasia and other related phenotypes: SEDC (c.1367G > C, p.Gly456Ala) ${ }^{27}$ and hypochondrogenesis (c.1376G > A, p.Gly459Asp). ${ }^{13}$ This may reflect (i) a differential clinical diagnosis, (ii) a different effect of the substitution upon the collagen molecule, or (iii) a subtle difference that the nucleotide change has on pre-mRNA processing. When comparing the different glycine substitutions in collagen molecules, and their resulting phenotypic outcome, the latter is often overlooked. It may be significant that the mutations p.Gly456Arg and p.Gly459Arg resulting in Kniest dysplasia are also predicted to subtly alter the splicing signals, whereas those resulting in SEDC (c.1367G > C, p.Gly456Ala) or hypochondrogenesis (c.1376G >A, p.Gly459Asp) do not.

Despite the fact that Kniest dysplasia is associated with dominant mutations, most reports in the literature describe simplex cases. Exceptions to this include (i) a pair of identical twins with Kniest dysplasia, ${ }^{7}$ (ii) a female patient with type 1 Stickler syndrome giving birth to a child with Kniest dysplasia, ${ }^{3}$ (iii) a male patient with a mild SEDC-like phenotype having a child with Kniest dysplasia, and (iv) subject KN1 (father with Stickler syndrome, child with Kniest dysplasia). ${ }^{9}$ This 'paradox' of a condition inherited in an autosomal dominant pattern manifesting clinically as sporadic disease with unaffected parents is also observed in other type I (for example, (MIM \#166210)) and II (for example, (MIM \#200610)) collagenopathies, and can be partially explained by de novo mutational events (somatic and germline mosaicism). ${ }^{9,28}$ Such biological phenomena have been demonstrated in many families with Kniest dysplasia (Table 2), and mosaicism has been demonstrated or presumed in at least four of the eight families in the present study (subjects KN1, KN3, KN4, and KN7). The recognition that parental mosaicism may be present in cases of Kniest dysplasia makes the recurrence risk estimation challenging. Recent advances in genomic technologies are expected to enhance both our understanding of the molecular pathology of this disorder and our ability to detect it prenatally.
The ophthalmopathy associated with Kniest dysplasia has similarities with the other type II collagenopathies 2,8 and is characterised by abnormal vitreous on slit lamp biomicroscopy, myopia, and a high risk of developing retinal detachment at a young age. The ophthalmologist is an important member of the multidisciplinary team required to manage these patients and regular ophthalmic examinations are recommended. Most COL2A1 variants associated with Kniest dysplasia are unlike classical loss-of-function mutations (Table 2) and cause a more severe phenotype than that of type I Stickler syndrome, which is often due to heterozygous nonsense or frameshifting COL2A1 changes. ${ }^{2,14}$ Therefore, it can be speculated that a dominant negative effect is exhibited in Kniest dysplasia with the product of the mutant allele not only being non-functional but also interfering with the function of the remaining normal allele. The frequency of de novo mutational events is high in this autosomal dominant disorder and this has implications with regards to genetic counselling.

\section{Summary}

What was known before

- Disorders of type II collagen including Kniest dysplasia and Stickler syndrome are often associated with a specific ophthalmopathy. Retinal detachment at a young age can be a feature of Kniest dysplasia and there is a need for ophthalmological assessment in all individuals with the disorder.

- Although Kniest dysplasia is inherited in an autosomal dominant pattern it can manifest clinically as sporadic disease. This can be partially explained by de novo mutations in COL2A1.

What this study adds

- Abnormal vitreous architecture is a consistent feature in molecularly confirmed cases of Kniest dysplasia.

- A case of Kniest dysplasia with bilateral retinal detachments in his twenties is discussed.

- The p.Gly483Glu mutation in COL2A1 can cause both Kniest dysplasia and spondyloepiphyseal dysplasia congenita. This suggests that current categorisation of type II collagenopathies often has no obvious mechanistic basis.

\section{Conflict of interest}

The authors declare no conflict of interest.

\section{Acknowledgements}

We particularly thank the family members for participating in this study. We are grateful to colleagues who referred patients to us at Addenbrooke's and to Wieslawa Johnson for her help with the assembly of the 
Kniest dysplasia panel. This work was supported in part by a grant from the University of Cambridge (Retinal Research Fund).

\section{References}

1 Bishop P. The biochemical structure of mammalian vitreous. Eye (Lond) 1996; 10(Pt 6): 664-670.

2 Snead MP, McNinch AM, Poulson AV, Bearcroft P, Silverman B, Gomersall $P$ et al. Stickler syndrome, ocular-only variants and a key diagnostic role for the ophthalmologist. Eye (Lond) 2011; 25: 1389-1400.

3 Winterpacht A, Hilbert M, Schwarze U, Mundlos S, Spranger J, Zabel BU. Kniest and Stickler dysplasia phenotypes caused by collagen type II gene (COL2A1) defect. Nat Genet 1993; 3: 323-326.

4 Kannu P, Bateman J, Savarirayan R. Clinical phenotypes associated with type II collagen mutations. J Paediatr Child Health 2012; 48: E38-E43.

5 Kniest W. [Differential diagnosis between dysostosis enchondralis and chondrodystrophy]. Z Kinderheilkd 1952; 70: 633-640.

6 Lachman RS, Rimoin DL, Hollister DW, Dorst JP, Siggers DC, McAlister W et al. The Kniest syndrome. Am J Roentgenol Radium Ther Nucl Med 1975; 123: 805-814.

7 Maumenee IH, Traboulsi EI. The ocular findings in Kniest dysplasia. Am J Ophthalmol 1985; 100: 155-160.

8 Meredith SP, Richards AJ, Bearcroft P, Pouson AV, Snead MP. Significant ocular findings are a feature of heritable bone dysplasias resulting from defects in type II collagen. Br J Ophthalmol 2007; 91: 1148-1151.

9 Nagendran S, Richards AJ, McNinch A, Sandford RN, Snead MP. Somatic mosaicism and the phenotypic expression of COL2A1 mutations. Am J Med Genet A 2012; 158A(5): 1204-1207.

10 Richards AJ, McNinch A, Martin H, Oakhill K, Rai H, Waller $\mathrm{S}$ et al. Stickler syndrome and the vitreous phenotype: mutations in COL2A1 and COL11A1. Hum Mutat 2010; 31: E1461-E1471.

11 Snead MP, Payne SJ, Barton DE, Yates JR, al-Imara L, Pope FM et al. Stickler syndrome: correlation between vitreoretinal phenotypes and linkage to COL $2 \mathrm{~A} 1$. Eye (Lond) 1994; 8(Pt 6): 609-614.

12 Fincham GS, Pasea L, Carroll C, McNinch AM, Poulson AV, Richards AJ et al. Prevention of retinal detachment in Stickler syndrome: the Cambridge prophylactic cryotherapy protocol. Ophthalmology 2014; 121: 1588-1597.

13 Nishimura G, Haga N, Kitoh H, Tanaka Y, Sonoda T, Kitamura $\mathrm{M}$ et al. The phenotypic spectrum of COL2A1 mutations. Hum Mutat 2005; 26: 36-43.

14 Nishimura G, Nakashima E, Mabuchi A, Shimamoto K, Shimamoto T, Shimao Y et al. Identification of COL2A1 mutations in platyspondylic skeletal dysplasia, Torrance type. J Med Genet 2004; 41: 75-79.

15 Spranger J, Winterpacht A, Zabel B. Kniest dysplasia: Dr. W. Kniest, his patient, the molecular defect. Am J Med Genet 1997; 69: 79-84.

16 Wilkin DJ, Artz AS, South S, Lachman RS, Rimoin DL, Wilcox WR et al. Small deletions in the type II collagen triple helix produce kniest dysplasia. Am J Med Genet 1999; 85: 105-112.

17 Weis MA, Wilkin DJ, Kim HJ, Wilcox WR, Lachman RS, Rimoin DL et al. Structurally abnormal type II collagen in a severe form of Kniest dysplasia caused by an exon 24 skipping mutation. J Biol Chem 1998; 273: 4761-4768.

18 Hicks J, De Jong A, Barrish J, Zhu SH, Popek E. Tracheomalacia in a neonate with Kniest dysplasia: histopathologic and ultrastructural features. Ultrastruct Pathol 2001; 25: 79-83.

19 Friede H, Matalon R, Harris V, Rosenthal IM. Craniofacial and mucopolysaccharide abnormalities in Kniest dysplasia. J Craniofac Genet Dev Biol 1985; 5: 267-276.

20 Al-Hashmi N, Imtiaz F, Ramzan K, Faden M, Shuaib T, Al-Otaibi L et al. Novel splice (IVS18 + 1G >C) mutation in COL2A1 causing Kniest dysplasia. Clin Dysmorphol 2013; 22: 39-41.

21 Yokoyama T, Nakatani S, Murakami A. A case of Kniest dysplasia with retinal detachment and the mutation analysis. Am J Ophthalmol 2003; 136: 1186-1188.

22 Chen L, Yang W, Cole WG. Recurrent transition at a CG dinucleotide in exon 12 of COL2A1 produces Kniest dysplasia with abnormal RNA splicing by chondrocytes and lymphoblasts and interruption of the triple helix of type II collagen. Ann N Y Acad Sci 1996; 785: 234-237.

23 Bogaert R, Wilkin D, Wilcox WR, Lachman R, Rimoin D, Cohn $\mathrm{DH}$ et al. Expression, in cartilage, of a 7-amino-acid deletion in type II collagen from two unrelated individuals with Kniest dysplasia. Am J Hum Genet 1994; 55: 1128-1136.

24 Cartegni L, Chew SL, Krainer AR. Listening to silence and understanding nonsense: exonic mutations that affect splicing. Nat Rev Genet 2002; 3: 285-298.

25 Cooper TA, Wan L, Dreyfuss G. RNA and disease. Cell 2009; 136: 777-793.

26 Tiller GE, Weis MA, Polumbo PA, Gruber HE, Rimoin DL, Cohn DH et al. An RNA-splicing mutation (G + 5IVS20) in the type II collagen gene (COL2A1) in a family with spondyloepiphyseal dysplasia congenita. Am J Hum Genet 1995; 56: 388-395.

27 Zhang Z, He JW, Fu WZ, Zhang CQ, Zhang ZL. Identification of three novel mutations in the COL2A1 gene in four unrelated Chinese families with spondyloepiphyseal dysplasia congenita. Biochem Biophys Res Commun 2011; 413: 504-508.

28 Biesecker LG, Spinner NB. A genomic view of mosaicism and human disease. Nat Rev Genet 2013; 14: 307-320.

29 Richards AJ, Snead MP. The influence of pre-mRNA splicing on phenotypic modification in Stickler's syndrome and other type II collagenopathies. Eye (Lond) 2008; 22: 1243-1250.

30 Chen L, Yang W, Cole WG. Alternative splicing of exon 12 of the COL2A1 gene interrupts the triple helix of type-II collagen in the Kniest form of spondyloepiphyseal dysplasia. J Orthop Res 1996; 14: 712-721.

31 Cole WG. Abnormal skeletal growth in Kniest dysplasia caused by type II collagen mutations. Clin Orthop Relat Res 1997; 341: 162-169.

32 Yang W, Cole WG. Low basal transcripts of the COL2A1 collagen gene from lymphoblasts show alternative splicing of exon 12 in the Kniest form of spondyloepiphyseal dysplasia. Hum Mutat 1998; Suppl 1: S1-S2.

33 Spranger J, Menger H, Mundlos S, Winterpacht A, Zabel B. Kniest dysplasia is caused by dominant collagen II (COL2A1) mutations: parental somatic mosaicism manifesting as Stickler phenotype and mild spondyloepiphyseal dysplasia. Pediatr Radiol 1994; 24 : 431-435. 
34 Desmet FO, Hamroun D, Lalande M, Collod-Beroud G, Claustres M, Beroud C. Human Splicing Finder: an online bioinformatics tool to predict splicing signals. Nucleic Acids Res 2009; 37: e67.

35 Wilkin DJ, Bogaert R, Lachman RS, Rimoin DL, Eyre DR, Cohn DH. A single amino acid substitution (G103D) in the type II collagen triple helix produces Kniest dysplasia. Hum Mol Genet 1994; 3: 1999-2003.

36 Fernandes RJ, Wilkin DJ, Weis MA, Wilcox WR, Cohn DH, Rimoin DL et al. Incorporation of structurally defective type II collagen into cartilage matrix in Kniest chondrodysplasia. Arch Biochem Biophys 1998; 355: 282-290.

37 Wada R, Sawai H, Nishimura G, Isono K, Minagawa K, Takenobu $\mathrm{T}$ et al. Prenatal diagnosis of Kniest dysplasia with three-dimensional helical computed tomography. J Matern Fetal Neonatal Med 2011; 24: 1181-1184.

38 Ridout CK, Moore DJ, Abbs S, Irving M. A new rapid service for COL2A1 or FGFR3 related skeletal dysplasias,
The Association for Clinical Cytogenetics and Clinical Molecular Genetics Society joint Spring Conference; 30 April-02 May 2012; Birmingham, UK.

39 Winterpacht A, Schwarze U, Mundlos S, Menger H, Spranger J, Zabel B. Alternative splicing as the result of a type II procollagen gene (COL2A1) mutation in a patient with Kniest dysplasia. Hum Mol Genet 1994; 3: 1891-1893.

40 Faber J, Winterpacht A, Zabel B, Eich G, Spycher MA, Briner J et al. Kniest dysplasia: clinical, pathologic, and molecular findings in a severely affected patient-and review of the literature. Fetal Pediatr Pathol 1998; 18: 187-206.

41 Winterpacht A, Superti-Furga A, Schwarze U, Stöss H, Steinmann B, Spranger J et al. The deletion of six amino acids at the C-terminus of the alpha 1 (II) chain causes overmodification of type II and type XI collagen: further evidence for the association between small deletions in COL2A1 and Kniest dysplasia. J Med Genet 1996; 33: 649-654. 